\title{
Anti-A and anti-B titers in pooled group 0 platelets are comparable to apheresis platelets
}

\author{
Laura L. Cooling, Theresa A. Downs, Suzanne H. Butch, and Robertson D. Davenport
}

BACKGROUND: Although uncommon, acute hemolytic transfusion reactions (AHTRs) have been reported after transfusion of group $\mathrm{O}$ single-donor apheresis platelets (SDPs) to group $A, B$, and $A B$ recipients. Current methods for identifying "high-titer" SDPs include tube and gel methods. The risk of a high-titer unit is considered low with group $\mathrm{O}$, poststorage, pooled platelet concentrates (PPLTs); however, data regarding anti-A and anti-B titers in PPLTs are lacking.

STUDY DESIGN AND METHODS: Anti-A and anti-B titers were determined in 185 PPLTs by direct agglutination using manual gel and tube methods. PPLTs tested included 124 group O PPLTs, 25 group A PPLTs, 26 group B PPLTs, and 10 PPLTs containing a mix of either groups $O$ plus $A$ or groups $O$ plus $B$ (mixed PPLTs). The reciprocal of the highest dilution giving macroscopic agglutination was considered the agglutinin titer.

RESULTS: Mean anti-A and anti- $B$ titers in group $O$ PPLTs were, respectively, 16 and 8 by tube and 64 and 32 by gel $(p<0.0001)$. Gel titers were one to two dilutions higher than tube and sensitive to reagent red cell lots. With the use of at least 64 as a critical titer, 60 percent of group O PPLTs tested by gel would be considered high-titer. In mixed PPLTs, the addition of one non-group O PLT significantly decreased or neutralized the corresponding anti-A or anti-B $(p<0.0001)$. CONCLUSION: Anti-A and anti-B titers in group $O$ PPLTs are comparable to those reported in group $O$ SDPs and significantly lower than titers reported in AHTR. A critical direct agglutinin titer of 64 for identifying high-titer units by gel is too low and should be increased to 128 or higher.
A

lthough relatively rare, acute hemolytic transfusion reactions (AHTRs) after out-of-group or plasma-incompatible platelet (PLT) transfusions continue to generate interest and discussion, with approximately 40 reported cases worldwide. ${ }^{1-3}$ In nearly all instances, the implicated units were singledonor apheresis platelets (SDPs) from donors possessing unusually high-titer anti-A and anti-B agglutinins. ${ }^{1,2}$ At present, the risk of an AHTR after an out-of-group PLT transfusion are unknown; however, facility-specific rates range from 1 in 2000 to less than 1 in 45,000..$^{4-7}$ In the United States, approximately 10 to 40 percent of all PLT transfusions are plasma-incompatible with the intended recipient. 1,6,7

In a recent survey of US hospitals, most transfusion services (83\%) have policies to avoid or minimize exposure of incompatible plasma to PLT recipients. ${ }^{2}$ Common strategies include policies to provide only ABO typespecific or plasma-compatible PLTs, volume reduction, and limitations in the quantity of incompatible plasma transfused over time., ${ }^{1,2}$ A small number of US institutions (2\%), as well as international blood centers, prospectively screen group O SDPs for the presence of high-titer agglutinins. ${ }^{1,2,8,9}$ These units are subsequently labeled as "high-titer" and are reserved for transfusion to group $\mathrm{O}$ recipients only. Group O SDPs are selectively screened

ABBREVIATIONS: AHTR(s) = acute hemolytic transfusion reaction(s); PPLT(s) = pooled platelet concentrate(s); $\mathrm{SDP}(\mathrm{s})=$ single-donor apheresis platelet(s); WBD-PLTs $=$ whole blood-derived platelet concentrates.

From the Department of Pathology, University of Michigan Hospitals, Ann Arbor, Michigan.

Address reprint requests to: Laura Cooling, MD, MS, Transfusion Medicine, University of Michigan Hospitals, 2F225 UH, Box 0054, 1500 E. Medical Center Drive, Ann Arbor, MI 481090054; e-mail: lcooling@med.umich.edu.

Received for publication February 26, 2008; revision received April 17, 2008, and accepted April 21, 2008.

doi: 10.1111/j.1537-2995.2008.01814.x

TRANSFUSION 2008;48:2106-2113. 
because of the 1) plasma volume per donor (200 to $400 \mathrm{~mL}$ ), 2) higher mean anti-A or anti-B titers in group $\mathrm{O}$ donors, and 3) overwhelming prevalence of group O SDPs among reported cases of PLT-associated AHTRs. ${ }^{1,2}$

Two major challenges facing standardized, prospective screening are the absence of a recognized reference method and critical end titer that will reasonably differentiate safe from high-titer SDPs without significantly hampering PLT availability to non-O patients. ${ }^{1}$ Methods currently employed include tube saline agglutination, with and without room temperature incubation or antiglobulin testing, microcolumn agglutination (gel), automated microplate technology, and assays for in vitro hemolysis. ${ }^{1,2}$ Critical titers range from more than 20 to more than 500 and are determined locally based on assay type. ${ }^{1}$ Recent studies with microcolumn or gel technology have reported a reasonable correlation between gel and routine tube titers, with minimal additional cost to a SDP ( $\$ 1.20$ per SDP). ${ }^{9}$ In the latter study, between 28 and 39 percent of group O SDP units tested were considered high-titer based on either a critical direct agglutinin titer of at least 64 (presumably immunoglobulin G [IgG]) or an indirect agglutinin titer of at least 256 (IgG) by gel.

Unlike SDPs, group O pooled PLT concentrates (PPLTs) are considered at minimal risk for precipitating an AHTR after a single out-of-group transfusion. PPLTs contain significantly less plasma per donor $(50-60 \mathrm{~mL})$, which is subsequently diluted four- to sixfold in the final pooled product. As a result, group O PPLTs should have a lower mean anti-A or anti-B agglutinin titer and a lower incidence of high-titer units than SDPs due to pooling of multiple donor plasma. Despite the widespread use of PPLTs for more than 40 years, little information is available regarding mean anti-A or anti-B agglutinin titers in PPLTs. In this study, we screened 185 PPLTs for anti-A and anti-B by direct agglutination using both standard tube and manual gel tests. Contrary to expectations, the distribution and mean agglutinin titers in PPLTs were comparable or higher than those reported in other studies of group $\mathrm{O}$ SDPs. ${ }^{9}$ In addition, our results suggest that published critical titers for identifying high-titer units by gel are too low and are susceptible to changes in reagent red cell (RBCs) lots.

\section{MATERIALS AND METHODS}

\section{PLT samples}

Leukoreduced, whole blood-derived PLT concentrates (WBD-PLTs) were obtained from the Southeast Michigan American Red Cross (Detroit, MI). For transfusion, 4 to 5 units of ABO type-specific, WBD-PLTs are pooled (plasma transfer set, CharterMedical Ltd, Winston-Salem, NC) immediately before transfusion by the hospital transfusion service. On occasion, more than one $A B O$ type (group O plus A; group O plus B) may be pooled for transfusion due to product shortages or to minimize wastage. For transfusion purposes, PPLTs containing at least one group O WBD-PLT unit are labeled and dispensed as group O. On average, the University of Michigan prepares and dispenses approximately 11,000 to 12,000 pools per year, representing a total of 55,000 to 60,000 WBD-PLTs.

Plasma samples for agglutinin titers were collected from the tubing of freshly prepared PPLTs. Tubing was stripped twice to assure that the contents were representative of the final pooled product and then heat-sealed to obtain a 10 - to $20-\mathrm{cm}$ sterile segment for testing. All segments were stored at $4^{\circ} \mathrm{C}$ and were tested within 2 weeks of collection. A clerical review was performed for all PPLTs tested to confirm the content and ABO type of each WBDPLT unit in the pool. A total of 185 PPLTs were tested including 124 group O, 25 group A, 26 group B, and 10 mixed PPLTs. Mixed PPLTs contained a mix of either group $\mathrm{O}$ and group $\mathrm{A}$ (5 PPLTs) or group $\mathrm{O}$ and group B (5 PPLTs). Approximately 95 percent (175/185) of PPLTs tested were composed of 5 units of WBD-PLTs: 10 PPLTs (5.4\%) contained only 4 units of WBD-PLTs.

\section{Anti-A and anti-B agglutinin titers}

Serial twofold dilutions of plasma were prepared in 0.9 percent saline using a calibrated pipette. Samples were tested, in parallel, for anti-A and anti-B by direct agglutination using both tube method and manual gel. Results were reported as the reciprocal of the last dilution demonstrating 1+ macroscopic agglutination. To avoid interexaminer variation during testing, all testing by tube method and manual gel were assigned and performed by the same individual throughout the study. Over the course of the study, two different lots of reagent RBCs were used for tube and gel testing.

Testing by tube method was performed by adding two drops of neat or diluted plasma and one drop of commercially prepared 3 percent pooled $A_{1}$ and B RBCs (Lots A660 and A690, Ortho Clinical Diagnostics, Raritan, NJ). Samples were centrifuged, without incubation, for 15 seconds (1290 relative centrifugal force). Results were read and recorded immediately after centrifugation. ${ }^{10}$

Anti-A and anti-B agglutinin testing by manual gel was performed per manufacturer's instructions. ${ }^{11}$ Specifically, $50 \mu \mathrm{L}$ of neat or diluted plasma and $50 \mu \mathrm{L}$ of commercially prepared $\mathrm{A}_{1}$ and B RBCs $(0.8 \%$; Lots $08 \mathrm{~A} 278$ and 08A293, Ortho Diagnostics) were added to buffered gel cards (Lot 012807004-02, MTS, Pompano Beach, FL) and then centrifuged (10 min, 90 relative centrifugal force). Results were read and recorded immediately after centrifugation. In addition, all gel cards were photocopied for permanent record keeping. 


\section{PLT transfusion response}

A retrospective chart review was performed for recipients of high-titer and mixed PPLT units. High-titer units for chart review were defined as an anti-A or anti-B titer of at least 128 by gel. Recipients of high-titer units were screened for evidence of hemolysis based on the recipients' $\mathrm{ABO}$ type and pre- and posttransfusion hemoglobin, lactate dehydrogenase, and bilirubin levels.

The PLT transfusion response was examined in recipients transfused with high-titer, ABO-incompatible PPLTs and mixed PPLTs. Because information necessary to calculate a corrected count increment was not available for many patients, an appropriate response to PLT transfusion was defined as an increase in the posttransfusion PLT count of $20 \times 10^{9}$ per L or greater at 1 to 4 hours after transfusion. Patients showing a poor response to a mixed PPLT transfusion were reviewed for evidence of recent PLT refractoriness in the 3 days before and after the transfusion in question. The latter included a review of all PLT transfusions within a 7-day period, patient diagnosis, ABO type, HLA antibody screen, and the presence of pertinent nonimmune factors (e.g., bleeding, disseminated intravascular coagulation, infection, splenomegaly) known to adversely affect PLT transfusion..$^{12,13}$

\section{Statistical analysis}

Results were plotted as the absolute number and relative percentage (\%) of units for each end titer. Histograms and $t$ tests were performed with commercial software (Kaleidograph, Synergy Software, Reading, PA). Chi-square tests were performed using software (EpiInfo, Centers for Disease Control and Prevention, Atlanta, GA).

\section{RESULTS}

\section{Anti-A and anti-B agglutinin titers in pooled group $O$ PLTs by tube method}

A total of 124 group O PPLTs were tested for anti-A and anti-B (Fig. 1A). By tube method, the direct agglutinin titer for anti-A and anti-B ranged from neat to 64 with mean titers of 16 and 8 , respectively. Only two group O PPLTs (1.6\%) had a titer of 64 or greater, which has been suggested as a critical direct agglutinin titer by gel. ${ }^{9}$ Anti-A and anti-B titers were also examined in 20 group O WBD-PLTs with similar results (data not shown). A subanalysis of 5-unit $(\mathrm{n}=114)$ and 4 -unit $(\mathrm{n}=10)$ PPLTs found no significant difference $(\mathrm{p}=0.95)$.

\section{Anti-A and anti-B agglutinin titers in pooled group O PLTs by gel method}

Anti-A and anti-B agglutinin titers were also determined, in parallel, by manual gel as originally described by
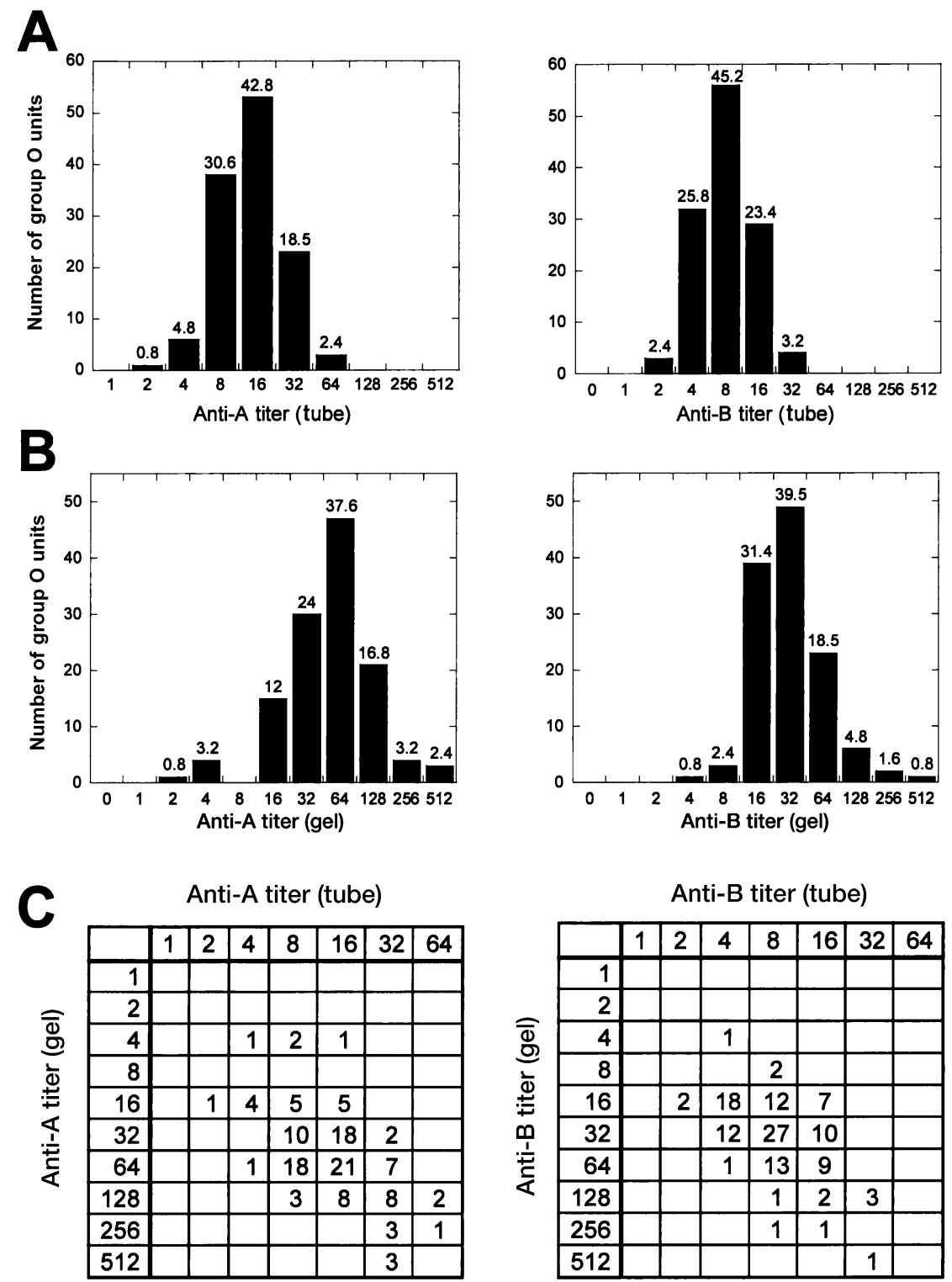

Fig. 1. Anti-A (left column) and anti-B (right column) titers in group O PPLTs. (A) Agglutinin titers by the tube method. (B) Agglutinin titers by the manual gel. Histograms show the number of PPLT units for each end titer. The relative percentage of units per end titer is denoted by the numbers atop each column. (C) Summary of tube and gel testing for each PPLT tested. Note that anti-B titers were available in only 123 of 124 group O PPLTs tested. 
Josephson and colleagues. ${ }^{9}$ As shown (Fig. 1B), anti-A ranged from 4 to 512 with a mean direct agglutinin titer of 64. Anti-B ranged from 4 to 512 with a mean titer of 32. In general, end titers were consistently one to two dilutions higher by gel than tube method (Fig. 1C, $p<0.0001$, paired $t$ test). With the use of at least 64 as a critical direct agglutinin titer, approximately 60 percent of group O PPLTs would be considered high-titer by gel. ${ }^{9}$ This is significantly higher than the percentage of high-titer units identified by tube ( $\mathrm{p}<0.00001$, chi-square).

\section{Anti-A titers in group B PPLTs}

We also examined anti-A direct agglutinin titers in 26 group B PPLTs (Fig. 2A). When compared to group O PPLTs, anti-A titers tended to be lower in group B PPLTs by one to three dilutions. Unlike group O PPLTs, there was no significant difference in end titer between gel and tube methods $(\mathrm{p}=0.38$ ). By both techniques, the mean anti-A titer was 8 . Anti-A titers were significantly decreased in group B PPLTs relative to group O PPLTs by gel ( 8 vs. $64, \mathrm{p}<0.0001$ ). No group B PPLTs had an anti-A titer of at least 64 .

\section{Anti-B titers in group A PPLTs}

Anti-B direct agglutinin titers were examined in 25 group A PPLTs (Fig. 2B). There was, on average, a higher mean titer by gel relative to tube ( 8 vs. $16, p<0.0001$ ). Overall, the anti-B titers in group A PPLTs were roughly the same as group O PPLTs by tube $(p=0.78)$. By gel, anti- $B$ titers were slightly decreased in group A PPLTs $(p=0.01)$. No group A PPLTs had an anti-B titer of 64 or greater.

\section{Anti-A and anti-B agglutinin titers in mixed PPLTs}

Although it is general policy to pool and dispense typespecific WBD-PLTs whenever possible, on occasion WBDPLTs of two ABO types were pooled (mixed PPLTs) for transfusion due to product shortages. PPLTs containing at least one group O WBD-PLT unit were labeled and dispensed as group $\mathrm{O}$. Over the 4-week course of the study, 10 units $(7.5 \%, 10 / 134)$ were tested that were, in fact, a mix of group $\mathrm{O}$ and non-OWBD-PLTs. These units were excluded from the group O PPLT data and analyzed separately.

As shown in Table 1, all mixed PPLTs were composed of either groups $\mathrm{O}$ plus $\mathrm{A}$ or groups $\mathrm{O}$ plus $\mathrm{B}$, with the number of non-O WBD-PLTs ranging from 1 to 4 units. Interestingly, the addition of even one non-O WBD-PLT unit (e.g., group B) markedly decreased or neutralized the corresponding isoagglutinin titer (e.g., anti-B). In 6 of 10 samples, no corresponding isohemagglutinin was identified by at least one method, even after testing with neat, undiluted plasma (Samples 86, 33, 128, 107,113 , and 118). When compared to group O PPLTs, the decrease in titers were highly significant $(p<0.0001)$. In contrast, titers of the uninvolved, remaining agglutinin (e.g., anti-A) were unchanged and consistent with group $\mathrm{O}$ PPLTs $(\mathrm{p}=0.28-0.42)$.

We also examined the clinical transfusion response to mixed PPLTs. Nine units were transfused to leukemia patients (9/10), including 7 patients with a history of hematopoietic progenitor cell transplant. In 9 patients, a 1- to 4-hour posttransfusion PLT count was available for analysis. As shown in Table 1, an appropriate increase in the posttransfusion count $\left(38.80 \times 10^{9} \pm\right.$ $14.72 \times 10^{9} / \mathrm{L}$; range, $23.00 \times 10^{9}$ to $55.00 \times 10^{9}$ ) was observed in 5 of 9 patients, regardless of recipient $\mathrm{ABO}$ type. Among the 4 patients with a poor response to transfusion, 3 had

Fig. 2. Anti-A and anti-B agglutinin titers in group B and group A PPLTs by tube (left column) and gel methods (right column). (A) Anti-A titers in group B PPLTs (四). (B) Anti-B titers in group A PPLTs $(\mathbb{I I})$. For reference, anti-A and anti-B titers in group $O$ PPLTs $(\square)$ are also shown. Histograms denote the percentage of units at each end titer. 


\begin{tabular}{|c|c|c|c|c|c|c|c|c|c|c|}
\hline \multirow[b]{3}{*}{ Sample } & \multicolumn{3}{|c|}{ Composition of mixed PPLT units ${ }^{*}$} & \multicolumn{4}{|c|}{ Agglutinin titer } & \multicolumn{3}{|c|}{ Transfusion response } \\
\hline & \multirow[b]{2}{*}{ Group O } & \multirow[b]{2}{*}{ Group A } & \multirow[b]{2}{*}{ Group B } & \multicolumn{2}{|c|}{ Anti-A } & \multicolumn{2}{|c|}{ Anti-B } & \multirow[b]{2}{*}{ Recipient $\mathrm{ABO}$} & \multirow[b]{2}{*}{ Clinical response† } & \multirow[b]{2}{*}{ PLT refractory } \\
\hline & & & & Tube & $\overline{\mathrm{Gel}}$ & Tube & Gel & & & \\
\hline 6 & 4 & 1 & & 1 & 2 & 8 & 16 & $\mathrm{O}$ & Yes & No \\
\hline 45 & 3 & 2 & & 1 & 2 & 2 & 8 & $\mathrm{O}$ & No & Yes \\
\hline 4 & 2 & 3 & & 2 & 8 & 8 & 32 & A & No & Yes \\
\hline 86 & 2 & 3 & & 0 & 1 & 2 & 16 & A & Yes & No \\
\hline 33 & 1 & 4 & & 0 & 0 & 8 & 32 & $\mathrm{O}$ & No & No \\
\hline 11 & 4 & & 1 & 8 & 64 & 1 & 4 & 0 & NA§ & Yes \\
\hline 128 & 4 & & 1 & 16 & 16 & 0 & 1 & $\mathrm{O}$ & No & Yes \\
\hline 107 & 3 & & 2 & 8 & 32 & 0 & 0 & $\mathrm{O}$ & Yes & No \\
\hline 113 & 3 & & 2 & 16 & 32 & 0 & 2 & A & Yes & No \\
\hline 118 & 2 & & 3 & 16 & 32 & 0 & 0 & A & Yes & No \\
\hline
\end{tabular}

laboratory evidence of clinical PLT refractoriness due to nonimmune factors (sepsis, disseminated intravascular coagulation, pulmonary hemorrhage)..$^{12,13}$ One group $\mathrm{O}$ patient, however, had an isolated, profound transfusion failure after transfusion of a mixed PPLT unit containing predominantly group A WBD-PLTs (Sample 33). The latter appears to be an example of ABO-associated PLT refractoriness in a group $\mathrm{O}$ recipient. ${ }^{1,14-17} \mathrm{An}$ anti-A titer of the recipient was not performed.

\section{Influence of reagent lots on end titers}

The majority of PPLTs were tested with a single lot of commercial reagent RBCs for tube and manual gel testing, respectively. However, a subset of 19 group O PPLTs (15\%) were tested at a later date, using a different RBC lot. To examine whether different reagent lots can influence end titer, we compared the mean end titer and distribution for each lot tested. Overall, there was no significant difference in mean titer between lots by tube testing. There was, however, a difference in testing by gel. Among the 19 PPLTs tested by gel using a different RBC reagent lot, the mean anti-A and anti-B titers were both lower (mean titer, 16; range, 4 to 128) than the population mean. Only 3 units (16\%) had a titer of 64 or greater (chi-square, $p<0.0001$ ).

An apparent decrease in gel titers was also noted when comparing tube and gel testing in paired samples (Fig. 1C). In the vast majority of group $\mathrm{O}$ samples tested (103/105, 98\%; RBC Lot 08A278), gel titers were always higher by at least one dilution over tube testing. In contrast, gel titers were the same or decreased relative to tube titers in 8 of $19(42 \%)$ samples tested with a different lot of 0.8 percent $A_{1}$ RBCs (Lot 08A293). These results suggest that anti-A and anti-B titers by gel may be more sensitive to changes in reagent lots than anticipated.

\section{Review of out-of-group PLT transfusions}

A retrospective review was performed for all group $\mathrm{O}$ PPLTs with an anti-A or anti-B titer of 128 or greater by gel.
A critical titer of 128 was chosen based on a review of European institutions, which use a critical titer between 100 to 200 for screening group $\mathrm{O}$ donors. ${ }^{1,8}$ Among group $\mathrm{O}$ PPLTs, 27 or 22 percent had a direct agglutinin titer of at least 128. As stated earlier, more than 60 percent of all group O PPLTs would be considered high-titer using a critical titer of 64 or greater as recommended by Josephson and colleagues. ${ }^{9}$

Among 27 high-titer group O PPLTs, 25 (93\%) were transfused to group $\mathrm{O}$ recipients. Two out-of-group transfusions (anti-A gel titer, 128) were transfused to group A recipients. There were no reports of transfusion reactions or laboratory evidence of hemolysis after transfusion. Both patients had an appropriate clinical response to transfusion based on a 1- to 4-hour posttransfusion PLT count (data not shown).

\section{Comparison of anti-A and anti-B titers in group 0 PPLTs and AHTRs}

To evaluate the potential risk of AHTRs due to group O PPLTs, we compared ABO titers in our group O PPLTs with published cases of AHTR (Fig. 3). Published cases were limited to AHTR after an out-of-group PLT transfusion, in which $\mathrm{ABO}$ titers were performed by direct agglutination using a standard tube method. A total of 23 published cases were identified for comparison. ${ }^{1-3}$ All cases selected involved transfusion of group O PLTs to an A or AB recipient. Results are presented as the percentage (\%) of units for each end titer (anti-A only). In PLT-associated AHTRs, anti-A titers ranged from 32 to 16,000 , with a mean titer of 512. In general, anti-A titers in group O PPLTs were significantly lower than those associated with AHTR.

\section{DISCUSSION}

In the past decade, there have been several reports of AHTR after transfusion of group O SDPs to group A, B, and 


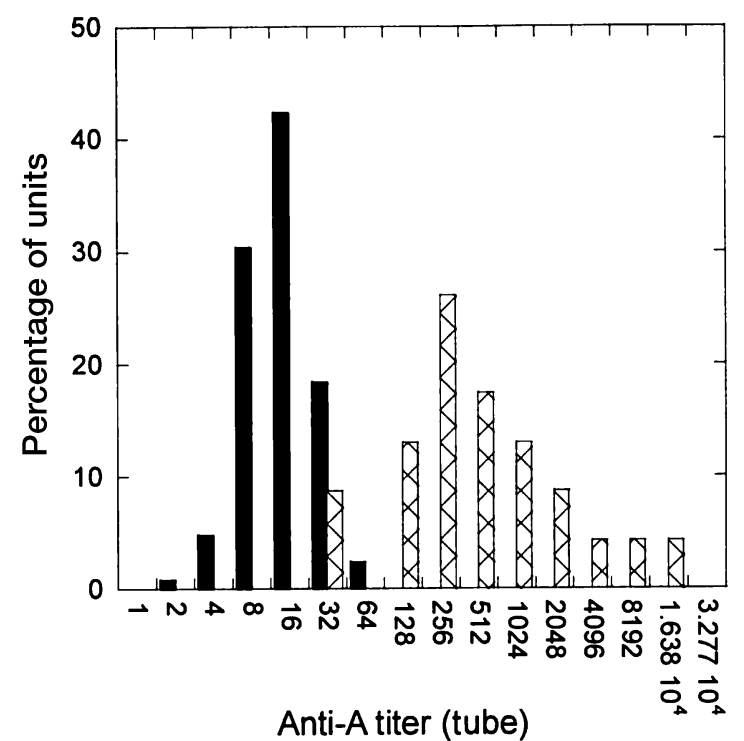

Fig. 3. Comparison of anti-A titers in group O PPLTs and PLTassociated AHTR. Anti-A tube titer reported in 23 published cases of AHTR after transfusion of group O PLTs ( $\otimes)$. As reference, anti-A tube titers in group $O$ PPLTs are also shown (ם).

$\mathrm{AB}$ recipients..$^{1-3,5-7}$ In nearly all cases, laboratory investigations revealed unusually high-titer anti-A or anti-B agglutinins in the donor. To reduce the risk of AHTR due to out-of-group SDP transfusions, several blood centers in Europe and the United States prospectively screen group O SDPs for high-titer donors. ${ }^{1,2,8,9}$ A variety of different testing platforms (tube, gel, microplate) and endpoints (direct agglutination, indirect antiglobulin test, hemolysis) are currently employed, with 3 to 39 percent of units testing as high-titer in recent studies. ${ }^{1}$

In 2004, Josephson and colleagues ${ }^{9}$ at Emory reported their experience using manual gel for screening group $\mathrm{O}$ SDPs. The gel method offers several potential advantages over tube testing including standardized pipetting of reagents and a stable agglutination reaction that may be reviewed and photographed or photocopied for record keeping. ${ }^{18,19}$ In their initial trial, the authors screened 100 group O SDPs for anti-A/AB over a period of 3 to 4 months, starting at an initial dilution of 32 . In their experience, 72 percent of units had a direct agglutinin titer of less than 64 , with a mean titer of 32 by gel. In 10 paired samples, gel titers were equivalent or one to two dilutions lower than tube titers. Equivalent or weaker agglutination with gel has been noted by others, particularly for anti-B. ${ }^{18,19}$ Based on their experience, the authors chose critical end titers of at least 64 for direct agglutination and at least 256 for indirect agglutination to identify high-titer units. In their pilot study, 28 percent of group O SDPs were classified as high-titer based on direct agglutination.

We sought to duplicate the findings of Josephson and coworkers with group $\mathrm{O}$, poststorage PPLTs. We anticipated that group O PPLTs would have a lower mean titer than that reported for group O SDPs with no hightiter units. Anti-A and anti-B agglutinin titers were determined, in parallel, by tube and gel methods in 185 PPLTs, including 124 group O PPLTs. Overall, the distribution and mean direct agglutinin titer ( 8 to 16) by tube method were consistent with recent studies in industrialized countries ${ }^{20,21}$ although significantly lower than older, frequently cited papers from the 1940s. ${ }^{22-24}$ Only 50 years ago, 13 to 65 percent of group $\mathrm{O}$ donors were considered "dangerous donors" based on tube titers of 256 or greater, with most donors (55\%-90\%) possessing titers of 64 or greater. ${ }^{22-24}$ The dramatic decrease in isoagglutinin titers observed in modern societies has been attributed to an increase in processed food. ${ }^{20}$

Relative to tube titers, direct agglutinin titers by gel were consistently higher by one to two dilutions in the majority of PPLTs tested. The exceptions were group B PPLTs and a subset of group $\mathrm{O}$ donors tested with a different lot of $A_{1}$ test cells. In these samples, equivalent anti-A titers were observed by tube and gel in 25 to 42 percent of units. Overall, the mean direct agglutinin titers in group $\mathrm{O}$ PPLTs were the same or higher than those reported for group O SDPs. ${ }^{9}$ More surprising, the percentage of group O PPLTs with direct agglutinin titers of at least 64 was significantly higher than group O SDP historical controls ( $60 \%$ vs. $28 \%, p=0.00002$ ). Unfortunately, we were unable to perform parallel testing in both PPLTs and SDPs due to the low numbers of SDPs available in our facility (10-20 per month).

Variation in commercial reagent RBCs may account for the unexpected number of high-titer units observed in our study. Although we used the same manufacturer as Josephson and colleagues for gel cards and reagent RBCs ( $0.8 \%$ suspension), our own data suggest that gel titers may be more sensitive than tube titers to different reagent RBC lots. Specifically, we noted a two- to fourfold difference in mean anti-A titers (16 to 64) between two-reagent $\mathrm{RBC}$ lots. This difference is comparable to that observed between our study and that by Josephson and colleagues, in which mean anti-A titers differed by a single dilution (64 vs. 32). It is interesting to note that the percentage of hightiter units in our study decreased from 60 to 22 percent by simply increasing the critical titer by one dilution (128). Our results indicate that variations in 0.8 percent RBCs can profoundly influence the percentage of units classified as high-titer at a direct agglutinin titer of 64 or greater.

In addition, we noted that direct agglutinin titers by gel tended to be higher than tube, which is contrary to the findings of Josephson and colleagues. This may reflect differences in RBC reagents, method, and number of samples tested by tube method between the two studies. Although we performed parallel testing in all samples, Josephson and coworkers ${ }^{9}$ performed gel and tube titers in only a small subset of samples $(n=10)$, which may have 
been insufficient to detect a difference between the two methods. In addition, the authors did not use commercial pooled reagent RBCs for tube testing but prepared their own 3 percent $A_{1}$ RBCs, suggesting the use of a single $A_{1}$ donor for testing. It has long been recognized that isoagglutinin titers can "vary with the same serum using different cells" 25 due, in part, to an almost 30 percent variation in the number of A antigen sites on $\mathrm{A}_{1} \mathrm{RBCs}^{26}{ }^{2}$ To minimize donor-specific variation, reagent manufacturers pool RBCs from two donors when preparing $A_{1}$ and $B$ reagent RBCs (personal communication, Ortho Clinical Diagnositics, Raritan, NJ). Finally, we performed tube testing by a standard "immediate-spin" technique whereas Josephson and colleagues included a 15-minute room temperature incubation before centrifugation, an established enhancement technique for isoagglutinin testing by tube. ${ }^{9,10}$

In addition to group O PPLTs, we serendipitously tested 10 mixed PPLTs, containing a mix of group $\mathrm{O}$ and either group A or group B WBD-PLTs. In general, the presence of even one non-O WBD-PLT unit significantly decreased or completely neutralized the corresponding agglutinin in group $\mathrm{O}$ plasma. We hypothesize that $\mathrm{ABO}-$ active substances present in plasma, and on PLTs, ${ }^{1,14}$ can bind and neutralize anti-A and anti-B agglutinins in group $\mathrm{O}$, mixed PPLTs. The ability of group $\mathrm{A}, \mathrm{B}$, and $\mathrm{AB}$ plasma to neutralize anti-A and anti-B agglutinins is well established and was the basis for "conditioned group O blood" and "universal plasma" in the early days of transfusion medicine. ${ }^{25,27}$ Pooled plasma preparations containing 5 to 50 units of plasma, regardless of donor ABO type, were transfused to adult trauma and surgical patients with few reported hemolytic transfusion reactions. ${ }^{25,28}$ Modern "pooled" universal plasma formulations (Uniplas, Octapharma Pharmazeutika, Austria; Bioplasma FDP, National Bioproducts Institute, Pinetown, South Africa) exist and contain a defined mixture of group $\mathrm{A}, \mathrm{B}, \mathrm{AB}$, and $\mathrm{O}$ donor plasma with isohemagglutinin titers of less than 8 to less than 32 and acceptable transfusion safety. ${ }^{29-31}$ Although extremely limited, our data suggest that mixed PPLTs, containing one WBD-PLT unit of recipient ABO type, could provide an additional layer of safety when out-of-group transfusion is unavoidable.

At present, poolingWBD-PLTs of different ABO types is generally discouraged due to possible immune complex formation, PLT activation, labeling requirements, and potential negative impact on the posttransfusion response. ${ }^{32-34}$ In times of shortages, however, it is sometimes necessary to pool WBD-PLTs of more than one ABO

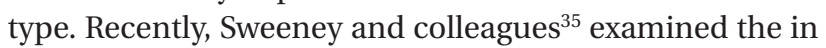
vitro effects of mixing group $\mathrm{O}$ and $\mathrm{A}$ WBD-PLTs using prestorage pooled PLTs (Acrodose, Pall Medical, Covina, CA). Over 5 days of storage, there was a small increase in P-selectin expression and PLT microaggregates in mixed PPLTs; however, there were no significant changes in PLT count, hypotonic shock response, shape change, glucose, lactate, pH, inflammatory cytokines, or coagulation factors. Although controlled clinical trials are lacking, anecdotal evidence suggests no increase in reported adverse reactions after transfusion of mixed PPLTs. ${ }^{36}$ Among our patients, most patients had an appropriate increase in PLT count with no reported adverse events. The single exception was a group $\mathrm{O}$ patient with evidence of ABO-incompatible PLT refractoriness after transfusion of a mixed PPLTs containing 4 units of group A WBD-PLTs. ${ }^{14-17}$

In summary, our findings show that group O PPLTs have anti-A and anti-B direct agglutinin titers comparable to those recently reported in group $\mathrm{O} \mathrm{SDPs}^{9}$ using both tube and gel methods. In addition, anti-A and anti-B agglutinin titers by gel are often higher than routine tube methods and may be particularly sensitive to changes in reagent $\mathrm{RBC}$ lots. Based on our data, we believe that a direct agglutinin titer of 64 or greater is too conservative and overly sensitive to reagent variability and could lead to substantial numbers of SDPs being unnecessarily labeled as high-titer.

These results also underscore the difficulty in establishing a reliable method and critical titer to identify high-titer PLTs. Ideally, the critical titer should identify units with clinically significant, high-titer anti-A or anti-B agglutinins with a reasonable risk of precipitating hemolysis after an out-of-group transfusion. Based on clinical reports, we have shown that 80 percent of all AHTRs had direct agglutinin titers of more than 128 . Based on the literature, we recommend increasing the critical direct agglutinin titer of 128 to 200 when using gel, which tended to have higher-end titers over the immediate-spin tube method in our experience. This would also be consistent with practices in the United States, England, and Sweden, which use direct agglutinin titers of more than 100 to more than 200 to identify hightiter units, with approximately 3 to 5 percent of donors testing as high-titer. The addition of screening with IgG gel cards may increase the number of high-titer SDPs identified by up to 10 percent, but may catch rare units with isolated elevated IgG titers missed by direct agglutination. .,37 $^{9,37}$

\section{REFERENCES}

1. Cooling L. ABO and platelet transfusion therapy. Immunohematology 2007;23:20-33.

2. Fung MK, Downes KA, Shulman IA. Transfusion of platelets containing ABO-incompatible plasma. Arch Pathol Lab Med 2007;131:909-16.

3. Harris SB, Josephson CD, Kost CB, Hillyer CD. Nonfatal intravascular hemolysis in a pediatric patient after transfusion of a platelet unit with high-titer anti-A. Transfusion 2007;47:1412-7.

4. Schwartz J, Depalma H, Kapoor K, Hamilton T, Grima K. 
Anti-A titers in group $\mathrm{O}$ single donor platelets: to titer or not to titer [abstract]. Transfusion 2003;43 Suppl:SP247.

5. Fauzie D, Shirey RS, Thoman S, Bensen-Kennedy D, King KE. The risk of hemolytic transfusion reactions due to passively-acquired $\mathrm{ABO}$ antibodies: a retrospective study of non-group $\mathrm{O}$ adult recipients of group $\mathrm{O}$ plateletpheresis transfusions [abstract]. Transfusion 2004;44 Suppl:SP10.

6. Mair B, Benson K. Evaluation of changes in hemoglobin levels associated with $\mathrm{ABO}$-incompatible plasma in apheresis platelets. Transfusion 1998;38:51-5.

7. Larsson LG, Welsh VJ, Ladd DJ. Acute intravascular hemolysis secondary to out-of-group platelet transfusion. Transfusion 2000;40:902-6.

8. Pietersz RN, Engelfriet CP, Reesink HW. International forum: transfusion of apheresis platelets and $\mathrm{ABO}$ groups. Vox Sang 2005;88:207-21.

9. Josephson CD, Mullis NC, Van Demark C, Hillyer CD. Significant numbers of apheresis-derived group $\mathrm{O}$ platelets have "high titer" anti-A/A,B: implications for transfusion policy. Transfusion 2004;44:805-8.

10. Brecher M. Technical manual. 15th ed. Bethesda (MD): American Association of Blood Banks; 2005.

11. Buffered MTS gel card (direction circular). Pompano Beach (FL): Micro Typing Systems; 1994.

12. Friedberg RC, Donnelly SF, Boyd JC, Gray LS, Mintz PD. Clinical and blood bank factors in the management of platelet refractoriness. Blood 1993;81:3428-34.

13. Slichter SJ, Davis K, Enright H, Braine H, Gernsheimer T, Kao KJ, Kickler T, Lee E, McFarland J, McCullough J, Rodey G, Schiffer CA, Woodson RW. Factors affecting posttransfusion platelet increments, platelet refractoriness, and platelet transfusion intervals in thrombocytopenic patients. Blood 2005;105:4106-14.

14. Cooling LL, Kelly K, Barton J, Hwang D, Koerner TA, Olson JD. Determinants of ABH expression on human blood platelets. Blood 2005;105:3356-64.

15. Jimenez TM, Patel SB, Pineda AA, Tefferi A, Owen WG. Factors that influence platelet recovery after transfusion: resolving donor quality from $\mathrm{ABO}$ compatibility. Transfusion 2003;43:328-34.

16. Heal JM, Blumberg N, Masel D. An evaluation of crossmatching, HLA, and ABO matching for platelet transfusions to refractory patients. Blood 1987;70:23-30.

17. Brand A, Sintnicolaas K, Claas FH, Eernisse JG. ABH antibodies causing platelet transfusion refractoriness. Transfusion 1986;26:463-6.

18. Langston MM, Procter JL, Cipolone KM, Stroncek DF. Evaluation of the gel system for $\mathrm{ABO}$ grouping and $\mathrm{D}$ typing. Transfusion 1999;39:300-5.

19. Kettler DS, Johnson MM. ABO grouping: experience with the gel test [abstract]. Transfusion 1997;37 Suppl:29S.

20. Mazda T, Yabe R, NaThalang O, Thammavong T, Tadokoro K. Differences in ABO antibody among blood donors: a comparison between past and present Japanese, Laotian, and Thai populations. Immunohematology 2007;23:38-41.
21. Saphire DG, Rudolph NS, Hackleman SM, Stone WH. The effect of age on the level of human ABO blood group antibodies. Aging Clin Exp Res 1993;5:177-84.

22. Aubert EF, Boorman KE, Dodd BE, Loutit JF. The universal donor with high titre iso-agglutinins: the effect of anti-A iso-agglutinins on recipients of group A. BMJ 1942;1:659-64.

23. Tisdall LH, Garland DM, Szanto PB, Hand AM, Bonnett JC. The effects of high isoagglutinin titer into recipients of other blood groups. Am J Clin Pathol 1946;16:193-206.

24. Ebert RV, Emerson CP. A clinical study of transfusion reactions: the hemolytic effect of group-O blood and pooled plasma containing incompatible isoagglutinins. J Clin Invest 1946 25:627-38.

25. Thalhimer W, Taylor ES. The low agglutinin titer of both small and large pools of plasma. JAMA 1945;128:277-9.

26. Beading W, Cooling LW. Immunohematology. In: McPherson RA, Pinkus MR, editors. Henry's clinical diagnosis and management by laboratory methods. 21st ed. Philadelphia (PA): WB Saunders; 2007. p. 619-69.

27. Klendshoj NC, Witebsky E. Transfusion of O blood conditioned by the addition of blood group specific substances. JAMA 1945;129:1091-3.

28. Miller EB, Tisdall LH. Reactions to 10,000 pooled liquid human plasma transfusions. JAMA 1945;128:863-7.

29. Solheim BG. Universal pathogen-reduced plasma in elective open-heart surgery and liver resection. Clin Med Res 2006;4:209-17.

30. Chapanduka ZC, Fernandes-Costa FJ, Rochat C, Blyth DF. Comparative safety and efficacy of Bioplasma FDP versus single-donor fresh-dried plasma in cardiopulmonary bypass patients. S Afr Med J 2002;92:356-7.

31. Rochat C, Larkin PM. Determination of proportion of $\mathrm{ABO}$ blood groups in manufacture of Bioplasma FDP: a universal plasma? [abstract]. Vox Sang 2006;91 Suppl 3:P474.

32. Heal JM, Masel D, Blumberg N. Interaction of platelet Fc and complement receptors with circulating immune complexes involving the ABO system. Vox Sang 1996;71:205-11.

33. Heal JM, Masel D, Rowe JM, Blumberg N. Circulating immune complexes involving the $\mathrm{ABO}$ system after platelet transfusion. Br J Haematol 1993;85:566-72.

34. Huang MM, Indik Z, Brass LF, Hoxie JA, Schreiber AD, Brugge JS. Activation of Fc $\gamma$ RII induces tyrosine phosphorylation of multiple proteins including FcyRII. J Biol Chem 1992;267:5467-73.

35. Sweeney J, Kouttab N, Holme ST, Cheves T, Nelson E. In vitro evaluation of prestorage pools consisting of mixed A and O platelet concentrates. Transfusion 2007;47:1154-61.

36. Policies on combining ABO non-identical platelet concentrates in the same pool. CBBS, e-network forum. Sacramento, CA: CBBS Central Office; 2004 Aug 22 [cited 2007 Feb 23]. Available from: http://www.cbbsweb.org/enf/ 2004/pltpool_mixabo.html.

37. Sadani DT, Urbaniak SJ, Bruce M, Tighes JE. Repeat ABOincompatible platelet transfusions leading to haemolytic transfusion reaction. Transfus Med 2006;16:375-9. 